\title{
AN ANALYSIS OF NEURAL ACTIVITY OF THE HUMAN BASAL GANGLIA IN DYSTONIA: A REVIEW
}

\author{
Ondřej Klempíř, Radim Krupička
}

\author{
Department of Biomedical Informatics, Faculty of Biomedical Engineering, Czech Technical \\ University in Prague, Kladno, Czech Republic
}

\begin{abstract}
Deep brain stimulation of the globus pallidus internus is an effective symptomatic treatment for pharmacoresistant dystonic syndromes, where pathophysiological mechanisms of action are not yet fully understood. The aim of this review article is to provide an overview of state-of-the-art approaches for processing microelectrode recordings in dystonia; in order to define biomarkers to identify patients who will benefit from clinical deep brain stimulation. For this purpose, the essential elements of microelectrode processing are examined. Next, we investigate a real example of spike sorting processing in this field. Herein, we describe baseline elements of microrecording processing including data collection, preprocessing phase, features computation, spike detection and sorting and finally, advanced spike train data analysis. This study will help readers acquire the necessary information about these elements and their associated techniques. Thus, this study is supposed to assist during identification and proposal of interesting clinical hypotheses in the field of single unit neuronal recordings in dystonia.
\end{abstract}

\section{Keywords}

electrophysiology, microrecording, spike sorting, biomarkers, neuromodulation, dystonia

\section{Introduction}

Electrophysiology is a means by which researchers are able to acquire neural electrical signals at various spatial scales. Several approaches can be used to study the nervous system-one of them is sensing action potentials on the surface of a nerve cell membrane using electrodes (Fig. 1). A lot of methods have been introduced to provide processing and analysis of neural signals. We primarily refer to single unit electrophysiology in dystonia, which is an important extrapyramidal study subject in neurology.

Dystonia is a movement disorder in which a person's muscles contract uncontrollably. The contraction causes the affected body part to twist involuntarily, resulting in repetitive movements or abnormal postures. Dystonia can affect one muscle, a muscle group, or the entire body. Dystonia affects about $1 \%$ of the population, and women are more prone to it than men [1]. Deep brain stimulation of the globus pallidus internus (GPi-DBS) is an effective treatment for pharmacoresistant dystonic syndromes, where pathophysiological mechanisms of action are not yet fully understood [2]. The age of onset is a factor in determining the phenotype of dystonia. The phenotype of childhood-onset dystonia is highly associated with the DYT1 mutation [3]. Whole exome sequencing is an efficient, sensitive and specific method for determining the genetic cause of dystonia [4].

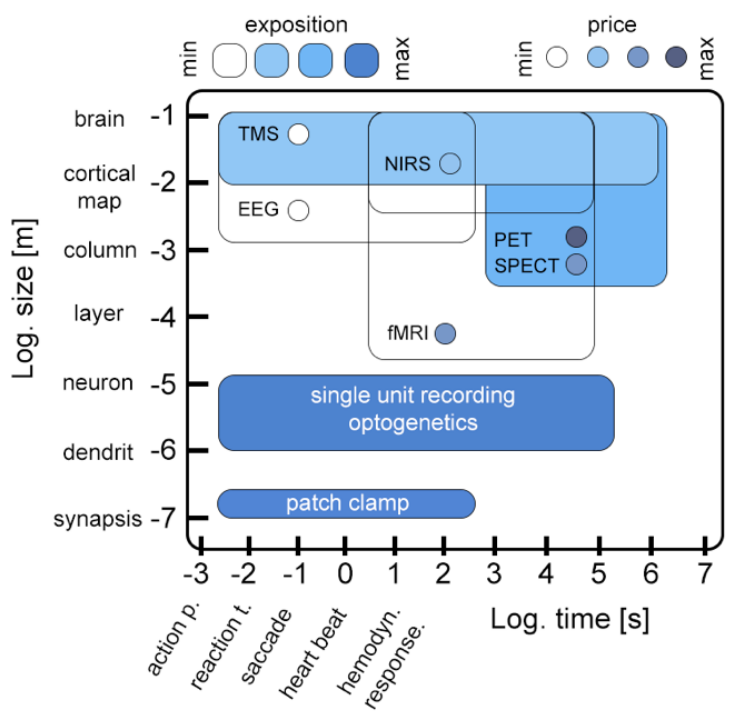

Fig. 1: Approximate ranges of spatiotemporal sensitivity of neural measurement techniques. Importantly, single unit approaches can measure and control a range of spatiotemporal organization relating to individual neurons and dendrites. Author's artwork created with inspiration from [5]. 
Although GPi-DBS is an effective treatment for dystonia, it may cause important stimulation-induced side-effects such as hypokinetic dysarthria or stuttering [6]. Noninvasive deep brain stimulation is an important goal in neuromodulation: a promising area of DBS in the future is the development of optogenetics. Chen et al. used specialized nanoparticles that can upconvert nearinfrared light from outside the brain into the local emission of blue light. They have injected these nanoparticles into a mouse brain and activated channel expressed in dopaminergic neurons with near-infrared light generated outside the skull at a distance of several millimeters [7].

This article reviews basic concepts and terminology from neural signal processing and spike statistics. It also describes the different types of neural signals, preprocessing, spike sorting, and lists available models with which these neural events are analyzed. Finally, it presents a real data example dealing with microrecording signal from the brain.

\section{Principles of measuring single unit neuronal activity}

There are two basic types of microelectrode recordings: intracellular and extracellular. Intracellular recordings form a group of techniques used to measure with precision the voltage across, or electrical currents passing through, neuronal or other cellular membranes by inserting an electrode inside the neuron [8]. Intracellular electrodes are made of thin glass pipettes that are pulled to a very fine and sharp ending or tip. The typical time course of the intracellular action potential with its first positive peak can be modelled by the Hodgkin-Huxley theory [9].

On the other hand, neuronal cell activity gives rise to transmembrane currents that can be measured in the extracellular medium. Although a major contributor of the extracellular signal is the synaptic transmembrane current, other sources-including $\mathrm{Na}^{+}$and $\mathrm{Ca}^{2+}$ spikes, ionic fluxes, ligand-gated channels and intrinsic membrane oscillations - can substantially shape the extracellular field. Electric fields can be monitored by extracellularly placed electrodes with submillisecond time resolution and can be used to interpret many facets of neuronal communication [10]. The shape of the extracellular action potential depends on the location of the electrodes and the experimental conditions. This phenomenon was illustrated on the recorded spikes from a 5-layer pyramidal cell with thin saline layer covering chip [11].

Recent advances in the development and application of nanoelectrodes enables simultaneous recording of intracellular and extracellular spontaneous spikes in mammalian neurons and cardiac cells [12]. A simultaneously recorded intracellular voltage gave the precise spike times of a local neuron, that can be used as the gold standard against which to compare the output of the extracellular spike detectors [13].

Extracellular neuronal activity is routinely explored in patients, using monopolar and multipolar microelectrodes in several parallel trajectories, during the implanttation procedure into the GPi with the aim of finding an optimal site for the placement of a permanent stimulation electrode. Presently, placement suitability is judged primarily by the neurologist, either visually or acoustically, based on a specific expression of neuronal action potentials (spikes). Due to their nature as all-ornone events, the occurrence of spikes over time is represented as a list of binary numbers (spike train), specifically zeroes for "no spike” or ones for a "spike”. Neurons use spikes as their preferred medium of neural coding communication. Because the physiological spike duration amounts to approx. $1.5 \mathrm{~ms}$, the signal should be sampled at units of $\mathrm{kHz}$.

\section{Analysis of extracellular neural activity recordings}

Electrophysiological microrecordings (MERs) are typically filtered in 500-5000 Hz. Bandpass filtering ensures the removal of slow shift and high frequency content while leaving the intermediate band (spiking) of frequencies intact. Spike sorting is considered the most important advanced processing step to accurately evaluate unit activity.

\section{Spike detection and sorting}

Data analysis for neuron recordings still requires two main problems to be solved - the reliable detection of spikes and the sorting of these spikes by their originating neurons. Finding the number of neurons is usually done manually during spike sorting-it is an unsupervised clustering problem where the number of the clusters is unknown. Approaches and solutions for both problems are difficult to evaluate quantitatively, due to a lack of knowledge about the truth behind the experimental data [14].

Spike sorting is one of the key techniques to understand brain activity. The process pipeline starts with a simple/adaptive threshold detection and ends with unsupervised sorting according to multidimensional feature extraction [15, 16] (Fig. 2). Machine learning techniques including deep learning, clustering, fuzzy, the Bayes approach and many more are successfully applied during spike sorting $[17,18]$.

Amplitude thresholding methods are the simplest: A spike is detected if the band pass filtered raw signals crosses a predefined threshold such as a multiple of the standard deviation of the underlying signal. A threshold that is frequently used is [19]: 


$$
T=n \text { median }\left(\frac{|x|}{0.6748}\right) \text {, }
$$

where $n$ is a constant (typically $n=4$ ) and the second term is an estimate of the standard deviation of the noise in the voltage $x$. Signal to Noise Ratio (SNR) is an important parameter that affects spike detection. The distance is often given in $\mathrm{dB}$, when the ratio is converted to ten times the decimal logarithm from the SNR.

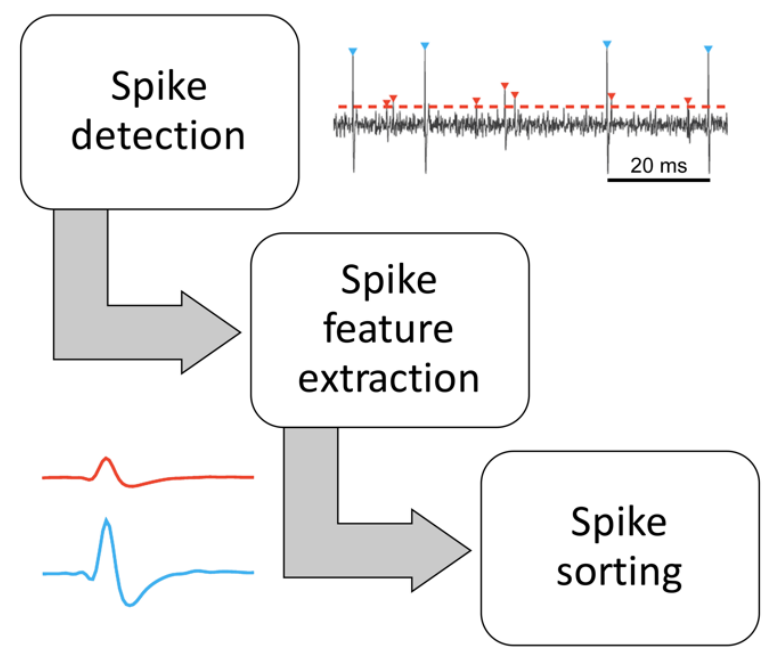

Fig. 2: Three principal stages of unsupervised spike sorting algorithms.

Usually, spike sorting analyzes are performed manually or with some semi-automatic tools, so it is a laborious and time-consuming task. Its objective automation is complicated. The purpose of various algorithms is to reduce the complexity and increase the accuracy of the entire process. Close neurons are first detected using an amplitude threshold. Peaks exceeding the threshold form a set of candidate spikes, which can then be clustered based on their amplitude and shape [16].

WaveClus algorithm by Quiroga has become a research milestone for the convenience and accuracy of sorting. Detected spikes can be described by many features: e.g. amplitudes, gradients in curves, WaveClus features, wavelets coefficients [20]. The best wavelet coefficients can be selected as those with the least Gaussian distribution, using a Kolmogorov-Smirnov test of normality.

The reduction of dimensionality, including the Principal Component Analysis (PCA) or Independent Component Analysis (ICA) [21], is a task employed prior to clustering. In a recent article, Dimitriadis et al. introduced the stochastic neighboring embedding (t-sne) dimensionality reduction method as a visualization tool in the spike sorting process. T-sne embeds the $\mathrm{n}$-dimensional extracellular spikes $(\mathrm{n}=$ number of features by which each spike is decomposed) into a low (usually 2D/3D) dimensional space. They show that such embeddings, even starting from different feature spaces, form obvious clusters of spikes that can easily be visualized and delineated with a high degree of precision [22].

An effective clustering algorithm relating to the WaveClus is superparamagnetic clustering. For instance, selected wavelet coefficients serve as the input for the unsupervised classification expectation maximization algorithm [23]. However, the use of various unsupervised approaches is available: K-means, Gaussian Mixture Models, DBSCAN etc. Links and comprehensive descriptions of publicly available code for spike sorting can be found in [24]. Examples of software packages and their mode of operation that have been used for human recordings are as follows:

- MClust - manual [25],

- KlustaKwik - semi-automatic with manual refinement [26],

- OSort - automatic online sorting, manual cluster selection [27],

- Wave_clus - automatic offline sorting, manual cluster selection [28].

\section{Spike train analysis}

Mathematically, spike train analysis is essentially an analysis of event-to-event data. Typically, the first step in spike data analysis involves visualizing and using simple descriptive statistics to characterize pertinent features of the data [29]. Various spike train metrics have been proposed to decode information content from biological spike trains [30]. Multineuron spike trains are called "parallel spike trains”. In the case of a single spike train, the main idea is to convert a spike train into a set of features that can be visualized and/or compared across different conditions, for instance:

- mean firing rate (MFR),

- instantaneous firing rate,

- variability,

- Fano factor,

- power spectra (and power in specific bands),

- inter-spikes interval (ISI),

- ISI histogram,

- coefficient of variation (CV) of ISI,

- autocorrelation,

- entropy,

- burst-based metrics: burst rate, number of spikes in a burst, inter-burst intervals, burst index,

- serial correlation,

- generalized linear model.

Basically, it is important to be able to characterize spike rhythms in GPi neurons and to know with statistical confidence when these rhythms have changed. E.g., a neurosurgeon hypothesizes that information relating to a movement task, such as planning versus the 
movement period and whether the movement is to the left or right, will influence this rhythmic spiking activity. The goal is to characterize spiking properties, identify whether rhythmic activity is present and statistically significant, and how such activity is influenced by the task variables. As with ISI models, maximum likelihood (ML) is performed to obtain general linear model (GLM) parameters. In general, it is not possible to obtain a closed form solution for the ML estimator. For this purpose, a numerical optimization technique such as Newton's method is suitable.

\section{Methods of assessing coupling between concurrent neuronal signals}

These methods are interested in quantitatively assessing the association between spike trains. Coupling among preprocessed signals can be essentially assessed by cross-frequency coupling (CFC) [31] and coherence [32]. Coherence is a measure of association between rhythms at the same frequency. Then, CFC focuses on assessing the association between different frequency rhythms. The Pearson's correlation coefficient is a less reliable but usable method. Generally, analysis of multiple parallel spike trains can be time consuming. The following lists highlight current methodological terms:

\section{Two spike trains}

- crosscorrelation,

- mutual information,

- Pearson correlation,

- Kullback-Leibler divergence,

- generalized linear models,

- coherence (partial coherence),

- comparison of instantaneous firing,

- joint pausiness.

\section{Many spike trains}

- snowflake plots,

- high-order correlations - CuBIC,

- latent dynamical approach,

- pairwise interaction,

- Hawkes models.

\section{Application to real data}

Many studies investigating neural activity have been performed in the GPi as well as in the subthalamic nucleus (STN). Recent technical and machine learning advances in microrecording have created new opportunities to study biomarkers related to various movement disorders, for instance, discovering an electrophysiological "sweet spot" for DBS-STN in Parkinson's disease [33]. Such studies aim to describe the functionality of specific neurons, which is of paramount importance in neuromodulation research with possible therapeutic applications in medicine. In this section we present a real example of signal processing according to the subset of the methods listed above.

For spike train analysis, spikes were extracted from a prototypical brain measurement with spontaneous spiking activity and then clustered using WaveClus [28] (Fig. 3). The recording time was $5 \mathrm{~min}$ at a sampling rate of $20 \mathrm{kHz}$.

Further, we applied generalized linear models in measuring the functional connectivity between a pair of neuronal spike trains, $x$ with times $s_{i}^{x}$ vs. another spike train $y$ with times $s_{i}^{y}$. Spike trains can be mathematiccally described as GLM point processes.

GLM is a class of models that generalizes the ordinary linear regression by allowing the predicted variable to have a distribution other than normal. To do this, the predicted variable is related to the regressors via a nonlinear link function. To fit a GLM to spike trains $x$ and $y$, the regressors have to be calculated using the spike trains and then GLM can be fitted using maximum likelihood optimization. After a model is obtained, its goodness-of-fit has to be investigated. Then, to find out whether a significant functional connection between $x$ and $y$ exists, the submodel of coefficients $\gamma$ is tested against the full model [34].

To characterize the level of communication between neurons, we evaluated timing relationships in spike trains of simultaneously recorded neurons. The result of the GLM fit is a set of coupling functions that estimate the change in the probability of a spike in one neuron in response to a spike from another simultaneously recorded neuron. For this spontaneous activity (Fig. 3a), GLMs did not evaluate the coupling as significant. To our knowledge, coupling investigation in the dystonic GPi-DBS connectomics has not been studied yet. The presented pipeline was implemented in the Matlab 2018b (MathWorks, USA) programming environment.

\section{Conclusion}

This review illustrates a practical approach for visualization of background firing activity from GPi neurons, computing and interpreting descriptive statistics including coupling, and building simple models of inter spike interval distributions. This review also presents the essential steps in processing real single-unit neural activity and extracting advanced statistics. 


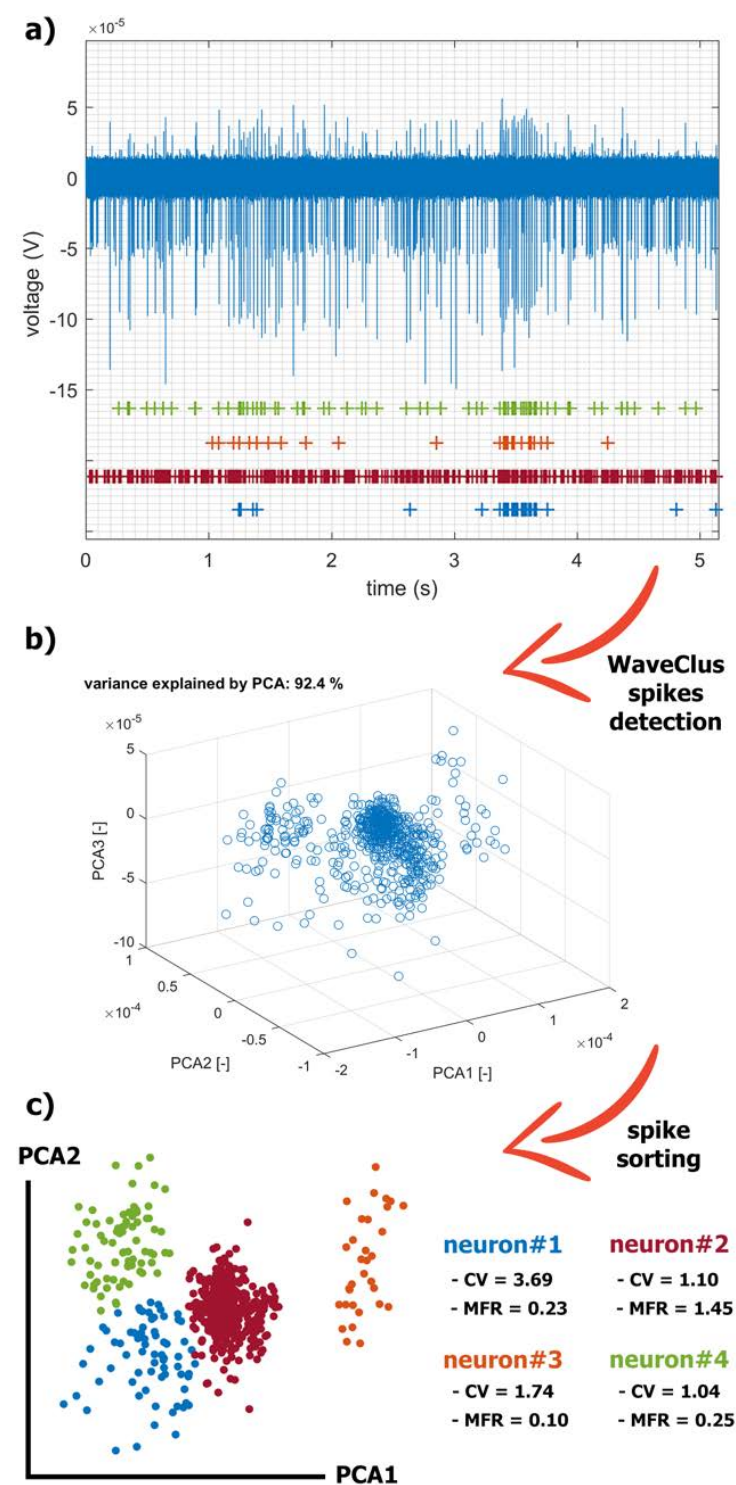

Fig. 3: The application of the selected methods for real $M E R$ processing. The recorded series is a summary activity of a large number of neurons further away from the electrode (background activity) and the activity of neurons in close vicinity of the electrode tip (single-unit activity). The signal can be decomposed in an estimation process called spike-sorting: a) A filtered raw signal and visualization of individual spike trains hidden in it. b) We used PCA to reduce multidimensional space after WaveClus detection. c) The spikes were sorted by the 4means method and characteristic statistics on extracted neurons are listed.

\section{Acknowledgements}

This study was supported by the Grant no. SGS19/090/OHK4/1T/17, Grant Agency of the Czech Technical University in Prague. We express thanks to co-supervisors, prof. Robert Jech and Tomáš Sieger,
Ph.D., for their knowledge support and scientific collaboration.

\section{References}

[1] Albanese A, Bhatia K, Bressman SB, Delong MR, Fahn S, Fung VS, Hallett M, Jankovic J, Jinnah HA, Klein C, Lang AE, Mink JW, Teller JK. Phenomenology and classification of dystonia: a consensus update. Mov Disord. 2013;28:863-73.

[2] Fečíková A, Jech R, Čejka V, Čapek V, Št’astná D, Štětkářová I. Benefits of pallidal stimulation in dystonia are linked to cerebellar volume and cortical inhibition. Scientific Reports. 2018;8(1). https://doi.org/10.1038/s41598-018-34880-z

[3] O’Riordan S, Raymond D, Lynch T, Saunders-Pullman R, Bressman SB, Daly L, Hutchinson M. Age at onset as a factor in determining the phenotype of primarytorsion dystonia. Neurology. 2004;63(8):1423-6. https://doi.org/10.1212/01.wnl.0000142035.26034.c2

[4] McInerney-Leo AM, Harris JE, Leo PJ, Marshall MS, Gardiner B, Kinning E, Leong HY, McKenzie F, Ong WP, Vodopiutz J, Wicking C, Brown MA, Zankl A, Duncan EL. Whole exome sequencing is an efficient, sensitive and specific method for determining the genetic cause of short-rib thoracic dystrophies. Clinical Genetics. 2015;88(6):550-7. https://doi.org/10.1111/cge.12550

[5] Kremláček J. Event related potentials: principles and practice. IUPESM 2018, Educational Session.

[6] Rusz J, Tykalová T, Fečíková A, Št’astná D, Urgošík D, Jech R. Dualistic effect of pallidal deep brain stimulation on motor speech disorders in dystonia. Brain Stimulation. 2018;11(4): 896-903. https://doi.org/10.1016/j.brs.2018.03.007

[7] Chen S, Weitemier AZ, Zeng X, He L, Wang X, Tao Y, Huang AJ, Hashimotodani Y, Kano M, Iwasaki H, Parajuli LK, Okabe S, Teh DB, All AH, Tsutsui-Kimura I, Tanaka KF, Liu X, McHugh TJ. Near-infrared deep brain stimulation via upconversion nanoparticle-mediated optogenetics. Science. 2018; 359(6376):679-84. https://doi.org/10.1126/science.aaq1144

[8] Weckstrom M. Intracellular recording. Scholarpedia. 2010;5(8): 2224. https://doi.org/10.4249/scholarpedia.2224

[9] Kumar J, Kumar J, Murali S, Bhakthavatchalu R. Design and implementation of Izhikevich, Hodgkin and Huxley spiking neuron models and their comparison. In 2016 International Conference on Advanced Communication Control and Computing Technologies (ICACCCT). IEEE. https://doi.org/10.1109/icaccct.2016.7831611

[10] Buzsáki G, Anastassiou CA, Koch C. The origin of extracellular fields and currents - EEG, ECoG, LFP and spikes. Nature Reviews Neuroscience. 2012;13(6):407-20. https://doi.org/10.1038/nrn3241

[11] Ness TV, Chintaluri C, Potworowski J, Łęski S, Głąska H, Wójcik DK, Einevoll GT. Modelling and Analysis of Electrical Potentials Recorded in Microelectrode Arrays (MEAs). Neuroinformatics. 2015;13(4):403-26. https://doi.org/10.1007/s12021-015-9265-6

[12] Dipalo M, Amin H, Lovato L, Moia F, Caprettini V, Messina GC, Tantussi F, Berdondini L, De Angelis F. Intracellular and Extracellular Recording of Spontaneous Action Potentials in Mammalian Neurons and Cardiac Cells with 3D Plasmonic Nanoelectrodes. Nano Letters. 2017;17(6):3932-9. https://doi.org/10.1021/acs.nanolett.7b01523

[13] Obeid I. Comparison of Spike Detectors based on Simultaneous Intracellular and Extracellular Recordings. In 2007 3rd International IEEE/EMBS Conference on Neural Engineering. IEEE. https://doi.org/10.1109/cne.2007.369696

[14] Mamlouk AM, Sharp H, Menne KM, Hofmann UG, Martinetz T. Unsupervised spike sorting with ICA and its evaluation using 
GENESIS simulations. Neurocomputing. 2005;65-6. https://doi.org/10.1016/j.neucom.2004.10.019

[15] Wild J, Prekopcsak Z, Sieger T, Novak D, Jech R. Performance comparison of extracellular spike sorting algorithms for singlechannel recordings. Journal of Neuroscience Methods. 2012; 203(2):369-76. https://doi.org/10.1016/j.jneumeth.2011.10.013

[16] Bakstein E. Deep Brain Recordings in Parkinson's Disease: Processing, Analysis and Fusion with Anatomical Models [dissertation]. Czech Technical University in Prague; 2016.

[17] Bestel R, Daus AW, Thielemann C. A novel automated spike sorting algorithm with adaptable feature extraction. Journal of Neuroscience Methods. 2012;211(1):168-78. https://doi.org/10.1016/j.jneumeth.2012.08.015

[18] Yang K, Wu H, Zeng Y. A Simple Deep Learning Method for Neuronal Spike Sorting. Journal of Physics: Conference Series. 910(1):12062. https://doi.org/10.1088/1742-6596/910/1/012062

[19] Ehrlich T. Single Neuron Studies of the Human Brain: Probing Cognition. Journal of Neuropathology \& Experimental Neurology. https://doi.org/10.1097/nen.0000000000000242

[20] Quiroga R. Spike sorting. Scholarpedia. 2007;2(12):3583. https://doi.org/10.4249/scholarpedia.3583

[21] Lopes MV, Aguiar E, Santana E, Santana E, Barros AK. ICA feature extraction for spike sorting of single-channel records. In 2013 ISSNIP Biosignals and Biorobotics Conference: Biosignals and Robotics for Better and Safer Living (BRC). IEEE. https://doi.org/10.1109/brc.2013.6487468

[22] Dimitriadis G, Neto J, Kampff A. T-SNE visualization of largescale neural recordings. Neural Comput. 2018;30(7):1750-1774. https://doi.org/ 10.1162/neco_a_01097

[23] Lourens MA, Meijer HG, Contarino MF, van den Munckhof P, Schuurman PR, van Gils SA, Bour LJ. Functional neuronal activity and connectivity within the subthalamic nucleus in Parkinson's disease. Clinical Neurophysiology. 2013;124(5): 967-81. https://doi.org/10.1016/j.clinph.2012.10.018

[24] Spike Sorting Software [online]. [cit. 2018-08-20]. http://simonster.github.io/SpikeSortingSoftware/

[25] Mclust Spike Sorting [online]. [cit. 2018-08-20]. http://redishlab.neuroscience.umn.edu/MClust/MClust.html

[26] KlustaKwik Spike Sorting [online]. [cit. 2018-08-20]. https://sourceforge.net/projects/klustakwik/

[27] OSort Spike Sorting [online]. [cit. 2018-08-20]. http://www.urut.ch/new/serendipity/index.php?/pages/osort.htm l

[28] WaveClus Spike Sorting [online]. [cit. 2018-08-20]. https://github.com/csn-le/wave_clus
[29] Kramer MA. Case Studies in Neural Data Analysis: A Guide for the Practicing Neuroscientist. Computational Neuroscience Series, 2016, The MIT Press. ISBN: 978-0262529372

[30] Tezuka T. Multineuron spike train analysis with R-convolution linear combination kernel. Neural Networks. 2018;102:67-77. https://doi.org/10.1016/j.neunet.2018.02.013

[31] Zhou P, Burton SD, Snyder AC, Smith MA, Urban NN, Kass RE. Establishing a Statistical Link between Network Oscillations and Neural Synchrony. PLOS Computational Biology. 2015;11(10): e1004549. https://doi.org/10.1371/journal.pcbi.1004549

[32] Hohlefeld FU, Huchzermeyer C, Huebl J, Schneider GH, Nolte G, Brücke C, Nikulin V. Functional and effective connectivity in subthalamic local field potential recordings of patients with Parkinson’s disease. Neuroscience. 2013;250:320-32. https://doi.org/10.1016/j.neuroscience.2013.07.028

[33] Horn A, Neumann WJ, Degen K, Schneider GH, Kühn AA. Toward an electrophysiological "sweet spot" for deep brain stimulation in the subthalamic nucleus. Human Brain Mapping. 2017. https://doi.org/10.1002/hbm.23594

[34] Vosmik J. Analysis of Parallel Microelectrode Recordings [master's thesis]. Czech Technical University in Prague; 2018. 